\title{
Estudio In Vitro de la resistencia al cizallamiento de una resina reforzada según el tratamiento del sustrato
}

In vitro study of the shear strength of a reinforced resin substrate by treating
Estudo in vitro da resistência ao cisalhamento de um substrato de resina reforçada por

tratamento
Fecha de Recepción

19 de febrero de 2016
Aceptado para su publicación

22 de abril de 2016
Angelina Rodríguez

Becaria de la Secretaria General de Ciencia y

Técnica UNNE. FOUNNE.

Email: angelinarodriguezI39@hotmail.com

Ángeles Pereyra Coimbra

Becaria de la Secretaria General de Ciencia y

Técnica UNNE. FOUNNE.

Email: angelespereyracoimbra@gmail.com

María Eugenia Zamudio Magister. Profesora Titular Cátedra de

Biomateriales. FOUNNE. Email:mezamudio@odn.unne.edu.ar

Nilda Alvarez Magister. Profesora Adjunta Cátedra de Biomateriales. FOUNNE. Email:nalvarez@odn.unne.edu.ar

Juan José Christiani

Doctor en Odontología. Docente Cátedra

Preclínica de Prótesis. FOUNNE. Email: jjchristiani@odn.unne.edu.ar

Lugar de Trabajo: Facultad de Odontología Universidad Nacional del Nordeste Av. Libertad 5450. CP.3400. Corrientes. Argentina

\section{Resumen}

El éxito de un tratamiento en odontología restauradora dependerá de la adhesión de la resina reforzada a la superficie dentaria. Para ello se tiene en cuenta las propiedades de las resinas, su composición de su relleno, el sistema adhesivo y otros factores tales como el tratamiento de sustrato de acuerdo al tipo de sustancia utilizada. Objetivo: Evaluar y determinar la resistencia mecánica de una resina reforzada de acuerdo al tratamiento previo del sustrato. Metodología: Se recolectó 20 piezas dentarias de bovinos, almacenadas en solución fisiológica a $37^{\circ} \mathrm{C}$. Fueron divididas en tres grupos teniendo en cuenta la profilaxis previa con: pasta fluorada, spray de bicarbonato, piedra pómez. Se realizó luego el mismo protocolo: técnica de grabado acido, sistema adhesivo, y resina reforzada para lo cual se utilizaron cilindros de acero de $4 \times 4$ $\mathrm{mm}$, colocados perpendicularmente sobre las superficies dentarias libres. La resistencia al cizallamiento se realizó con la máquina de ensayo universal Instron ${ }^{\circledR}$. Resultados: El grupo donde se utilizó como profilaxis spray de bicarbonato presentó mayor resistencia al cizallamiento; 4,63 \pm DS I, I8, mejorando la adhesión con valores estadísticamente significativos respecto a los otros grupos. Conclusiones: la resina reforzada previo tratamiento del sustrato con bicarbonato presenta mayor fuerza de unión en la interfase adhesivo-superficie dentaria. 
REVISTA FACULTAD DE ODONTOLOGÍA

ISSN No 1668-7280 - Vol. IX No 1 - 2016

8
INVESTIGACIÓN

Rodríguez - Pereyra Coimbra - Zamudio - Alvarez - Christiani
Palabras claves

Resina reforzada, adhesivo, profilaxis, cizallamiento.

\section{Abstrac}

The success of a restorative dentistry treatment will depend on the accession of the reinforced resin to the tooth surface. For this takes into account the properties of the resins, the composition of the filler, the adhesive system and other factors such as the substrate processing according to the type of substance used. Objective: To evaluate and determine the strength of enhanced according to the resin substrate pretreatment. Methods: 20 bovine teeth, stored in saline solution at $37{ }^{\circ} \mathrm{C}$ was collected. They were divided into three groups formed taking into account the previous prophylaxis: fluoride toothpaste, baking spray, pumice. Acid etching technique, adhesive system, and for which reinforced resin steel cylinders $4 \times 4 \mathrm{~mm}$ were used, placed perpendicularly on the free tooth surfaces: The same protocol was then performed. The shear strength was performed with Instron ${ }^{\circledR}$ machine universal testing. Results: Group where he was used as a spray of bicarbonate prophylaxis had higher shear strength; DS $4.63 \pm \mathrm{I} .18$, improving adhesion with statistically significant values compared to the other groups. Conclusions: Pre reinforced resin substrate with bicarbonate treatment has greater bonding strength in the adhesive-tooth interface surface.

\section{Keywords}

Reinforced resin, adhesive, prevention, shearing.

\section{Resumo}

O sucesso de um tratamento odontologia restauradora vai depender da adesão da resina reforçada a superfície do dente. Para isso leva em conta as propriedades das resinas, a composição do material de enchimento, o sistema adesivo e outros factores, tais como o processamento de substrato de acordo com o tipo de substância utilizada. Objectivo: Avaliar e determinar a resistência melhorada de acordo com o pré-tratamento do substrato de resina. Métodos: 20 dentes bovinos, armazenados em solução salina a $37{ }^{\circ} \mathrm{C}$ foi recolhido. Eles foram divididos em três grupos formados, tendo em conta a profilaxia anterior: creme dental com flúor, spray de cozimento, pedra-pomes. Condicionamento ácido sistema adesivo, e para os quais cilindros de aço reforçado de resina foram utilizados $4 \times 4$ $\mathrm{mm}$, colocados perpendicularmente sobre as superfícies dos dentes livres: $O$ mesmo protocolo foi então realizada. A resistência ao cisalhamento foi realizada com a máquina universal de ensaios Instron ${ }^{\circledR}$. Resultados: Grupo onde ele foi usado como um spray de profilaxia bicarbonato teve maior força de cisalhamento; DS 4,63 $\pm 1,18$, melhorando a adesão com valores estatisticamente significativos em comparação com os outros grupos. Conclusões: Pré reforçado substrato de resina com bicarbonato de tratamento tem maior força de ligação na superfície interface adesivo-dente.

\section{Palavras chave}

Resina reforçada, adesivo, prevenção, tosquia.

\section{Introducción}

Los materiales de restauración más utilizados en la actualidad son las resinas reforzadas, tanto en el sector anterior como en sector posterior de la cavidad bucal. La demanda progresiva de estética derivó en el aumento gradual de estudios y marcas comerciales tanto de las resinas reforzadas, como así también de los sistemas adhesivos buscando mejorar los mismos ${ }^{1,2,3}$.

Durante mucho tiempo, las resinas reforzadas, fueron materiales de elección para el sector anterior exclusivamente, esta situación ha sido revertida ya que actualmente estos materiales son superiores respecto a propiedades mecánicas, situación que beneficia a las indicaciones de uso, siendo estas cada vez más extensas. La selección del material a utilizar transcurre entre los requerimientos mecánicos y los estéticos deseados, si se necesitan buenas propiedades mecánicas se seleccionan materiales que contienen mayor cantidad de relleno, mientras que si el requerimiento tiene una fuerte necesidad de estética se seleccionará una resina con el mínimo de tamaño de partícula. El avance de estos materiales sigue evolucionando lo que hace necesario conocer continuamente el tipo de material a seleccionar 
en cada caso clínico como así también la técnica o el sistema adhesivo a utilizar ${ }^{1,2,3}$.

Así mismo es importante conocer que un punto crítico a tener en cuenta es la unión superficie dentaria con el material restaurador. Para ello se debe realizar un correcto acondicionamiento del sustrato para lograr una correcta adhesión y en consecuencia el éxito clínico. Se sabe que los depósitos de la superficie dental pueden eliminarse por varios métodos: copas o cepillos rotatorios con pastas abrasivas, o bien puntas ultrasónicas. Todos estos métodos se basan en el principio de adaptar cristales abrasivos o impulsos ultrasónicos a la superficie que debe pulirse. El método más tradicional consiste en una mezcla de polvo de piedra pómez y agua, pero se ha observado que utilizando piedra pómez pueden quedar restos de la misma que interfieran en el proceso del grabado ácido ${ }^{4,5,6}$.

Desde el punto de vista del proceso adhesivo, alteraciones en el substrato, pueden determinar disminución en la resistencia de adhesión del material restaurador, aumentar el grado de filtración marginal y consecuentemente, comprometer la longevidad de la restauración ${ }^{7}$.

Las razones antes mencionadas deben ser atendidas al momento de realizar una restauración en la cavidad bucal, revisten suma importancia: el tratamiento del sustrato, la selección del sistema adhesivo y la resina escogida. El punto de inflexión en la utilización de las resinas en la odontología restauradora lo representa la unión entre el tejido dentario y el material restaurador, ello exige la conformación de una sola estructura para realizar una práctica odontológica conservadora. La adhesión fue el descubrimiento más importante de la odontología en la historia, ya que surgió como panacea de las restauraciones que fracasaban por la filtración marginal al no existir una interrelación entre estructura dentaria y material restaurador.

Los sistemas adhesivos al igual que los composites han sobrellevado un desarrollo tecnológico progresivo en los últimos años, constituyen en la actualidad los biomateriales más analizados por los investigadores, ya que crean el conjunto del que depende la mayoría de los procedimientos restauradores relacionados con la conservación y estética dentaria.
Es importante destacar que la mayoría de las investigaciones que se desarrollan en el ámbito mundial tienen como finalidad investigar:

- Los nuevos sistemas adhesivos para sintetizar los pasos clínicos alegando que al desarrollar menos pasos clínicos se disminuyen las causas de fracaso en las restauraciones con resinas reforzadas. Los trabajos comparan in Vitro los sistemas adhesivos con menor número de pasos clínicos, aunque esta simplificación de las fases clínicas (técnica de adhesión) no signifiquen en los resultados encontrados, necesariamente mejoras en la fuerza de adhesión (Mpa) o una disminución importante del tiempo total de trabajo.

- La microfiltración, medición de grosor de película en adhesivos, fuerzas de tracción, de desalojo, de micro tracción, comparación de la resistencia adhesiva utilizando diferentes sistemas adhesivos, diferentes resinas $y$ diferentes protocolos de grabado acido.

En relación al proceso de adhesión, se establece que es un fenómeno complejo, que sufre la influencia directa de las propiedades físicoquímicas del adhesivo y del adherente ${ }^{8}$.

A sí mismo, la literatura da cuenta de trabajos que demuestran que la fuerza de adhesión entre la superficie del esmalte y la resina disminuye drásticamente cuando se utiliza la técnica de microabrasión sin acondicionamiento de la superficie, mientras que es un procedimiento eficaz para conseguir adhesión al esmalte por sí misma.

Otros autores señalan que la discrepancia de resultados puede estar en parte relacionada a las limitaciones de los test comúnmente aplicados para evaluar la interfase adhesiva ${ }^{9,10}$.

Las mismas discrepancias se observan cuando se habla de la realización de profilaxis al esmalte dentario, si bien todos los autores refuerzan la idea de que la profilaxis es fundamental cuando se habla de adhesión, todos señalan diferencias entre el tipo de tratamiento a realizar: piedra pómez y agua, pastas fluoradas, spray con bicarbonato 8,9,10.

Es fundamental resaltar que uno de los puntos importantes a la hora de comparar los resultados de la fuerza de adhesión entre los diferentes estudios, es valorar el tipo de prueba de fuerza que ha sido utilizado. Esta puede ser de resistencia al corte, tracción, flexión o una combinación de ambas. 
El objetivo fue evaluar y determinar la resistencia mecánica de cizallamiento de una resina reforzada de acuerdo al tratamiento previo del sustrato.

\section{Materiales y Métodos}

Se realizó un estudio experimental in vitro. Se recolectaran 20 piezas dentarias de bovinos, posteriormente se almacenaron en solución fisiológica a una temperatura de $37{ }^{\circ} \mathrm{C}$ durante una semana. Se procedió a la eliminación de los restos de encía y ligamento periodontal con bisturí y posteriormente se realizó la desinfección de los mismos con timol; se enjuagaron con agua destilada y se realizó la inclusión en cilindros con resinas acrílicas de autocurado, teniendo la precaución de que la cara vestibular sobresalga por encima de los cilindros.

Se dividieron las piezas dentarias en tres grupos de 10 especímenes cada uno y se procedió con el tratamiento del sustrato:

- Grupo I: Protocolo habitual profilaxis con pasta fluorada - grabado acido- sistemas adhesivos- resinas.

- Grupo 2: Protocolo con spray de bicarbonato grabado acido- sistemas adhesivosresinas.

- Grupo 3: Protocolo habitual profilaxis con piedra pómez - grabado acido- sistemas adhesivos- resinas.

Para la profilaxis del grupo I y 2 se utilizó un cepillo en forma de copa con micromotor a baja velocidad. Para el grupo 3 se utilizó un aeropulidor que actúa expulsando sobre la superficie dental, un chorro controlado de partículas muy pequeñas abrasivas de bicarbonato de sodio, suspendido en una aspersión de agua.

Para estandarizar el área de trabajo: técnica de grabado acido, sistema adhesivo y de empaquetamiento de la resina reforzada, se utilizaron cilindros de acero de $4 \times 4 \mathrm{~mm}$, para ser colocados perpendicularmente sobre las superficies dentarias libres (vestibular).

La aplicación del sistema adhesivo se realizó siguiendo las indicaciones del fabricante.

Se utilizó la misma resina-sistema adhesivo y ácido para cada uno de los grupos (Gel ácido al $37 \% 3 \mathrm{M} \circledast$, ZI $003 \mathrm{M} \circledast$, - Single Bond $3 \mathrm{M} \AA$ ).
La resina fue llevada en forma incremental en los cilindros de acero antes mencionados posicionados sobre la superficie vestibular de la pieza dentaria. La polimerización se realizó con lámpara de luz halógena, a una distancia de $1 \mathrm{~mm}$ durante 40 segundos cada capa.

Posteriormente los especímenes fueron almacenados en agua destilada a $37^{\circ} \mathrm{C}$ por 24 hs para luego ser llevadas a una máquina universal de ensayos Instron modelo 3366 (Figura I), donde se realizó fuerzas de cizalla (Figura 2), bajo una carga constante de cizallamiento de $100 \mathrm{KgF}$ y a una velocidad de cabezal de I mm por minuto, registrando los datos en mgapascales.

Los datos obtenidos fueron analizados mediante análisis de varianza (ANOVA) y las medias se compararon con el test de Tuckey a un nivel de significancia de 0,05 . Se utilizó el programa estadístico Infostat (versión 20I3).

\section{Resultados}

En el gráfico I se puede observar como el grupo donde se empleó resina reforzada con profilaxis con protocolo de tratamiento con Spray de Bicarbonato presentó mayor resistencia al cizallamiento respecto a los otros grupos, siendo el de menor valor el tratamiento con profilaxis con piedra pómez.

En el cuadro I se puede observar mediante el análisis del Test de Tukey, donde el protocolo donde se utilizo spray de bicarbonato se observó diferencia estadísticamente significativa que el resto de los grupos.

El cuadro nos indica las medias con una letra común que la diferencia no es estadísticamente significativa $(p>0,05)$.

\section{Discusión}

Diversos autores refieren respecto a los distintos métodos de profilaxis afirmando que no existen diferencias en cuanto a fuerzas de adhesión se refiere, entre realizar la profilaxis previa del esmalte con pastas fluoradas o no. Sin embargo Aboush" y col en un trabajo similar observaron una mayor resistencia en el grupo tratado con pasta fluorada.

Ray Bogert y García-Godoy ${ }^{12}$ realizaron un estudio de cizallamiento con pastas con o sin flúor, 


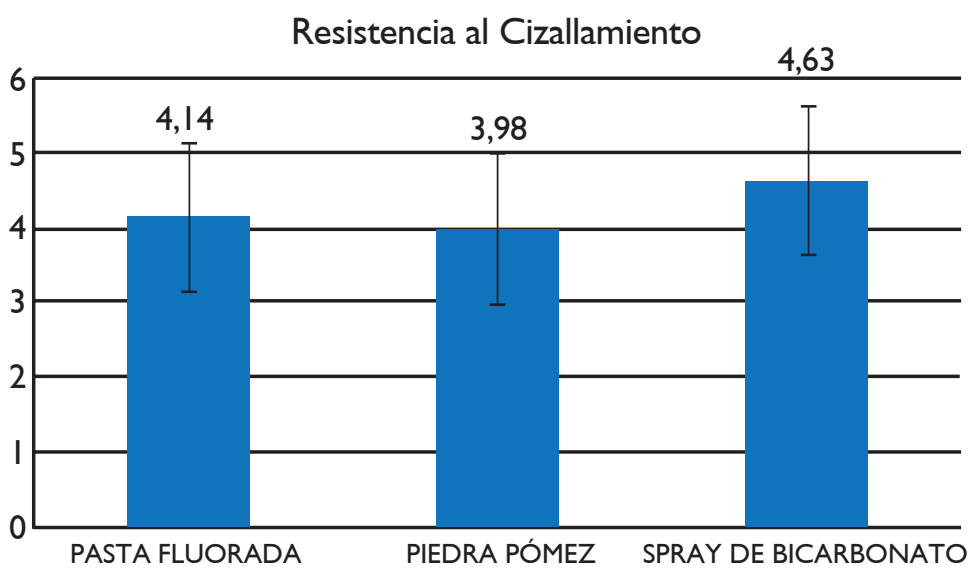

Gráfico I. Resistencia al Cizallamiento de Resina reforzada según el tratamiento previo.

Cuadro I. Resistencia al Cizallamiento de Resina reforzada según el tratamiento previo.

\begin{tabular}{|c|c|c|}
\hline Grupos & $\mathrm{n}$ & Medias \\
\hline I & 10 & 4,14 \\
\hline II & 10 & 3,98 \\
\hline III & 10 & 4,63 \\
\hline
\end{tabular}

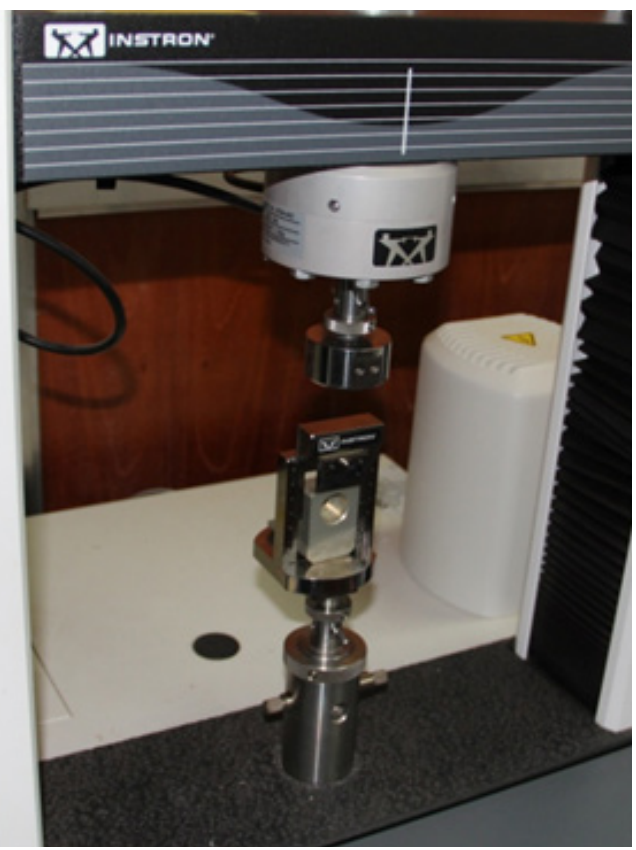

Figura I. Maquina universal de ensayos Instron modelo 3366. que además compararon con la profilaxis clásica con pasta de piedra pómez y con un grupo control que sólo se limpió con agua; observaron que ninguno de estos métodos influía en las fuerzas de adhesión de selladores.

Brown $^{13}$ y col hallaron mejores resultados con valores significativos similar al encontrado en este estudio, con la utilización del pulido con aire, bien sea con partículas de óxido de aluminio o de bicarbonato sódico.

Estudios demostraron ${ }^{13}$ de forma significativa una mayor resistencia utilizando como profilaxis pasta de profilaxis fluorada y spray de bicarbo-

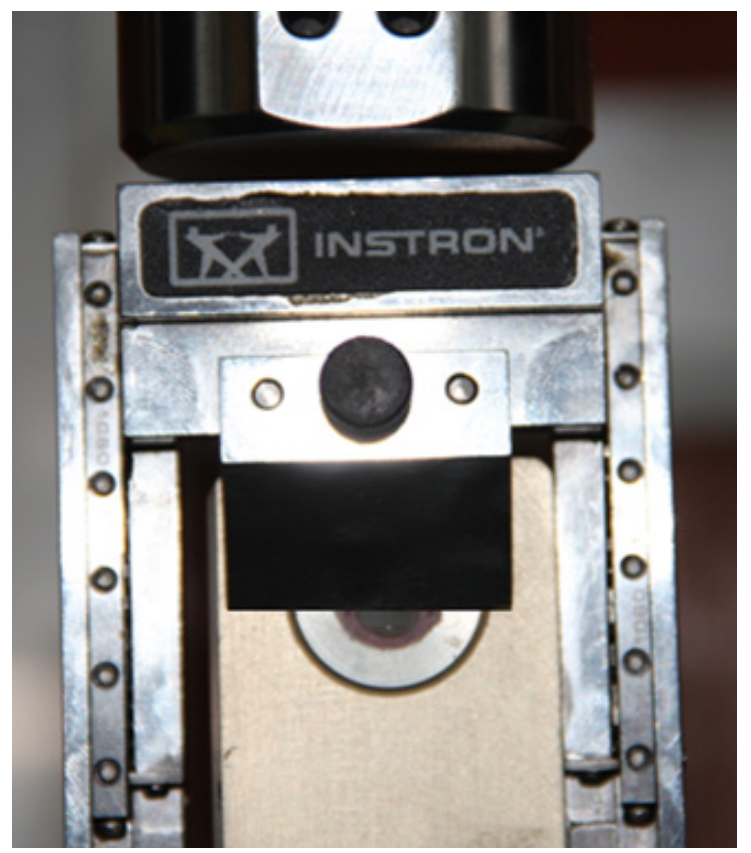

Figura 2. Prueba de cizalla con la máquina universal de ensayo Instron. 
REVISTA FACULTAD DE ODONTOLOGÍA

ISSN No 1668-7280 - Vol. IX No 1 - 2016
INVESTIGACIÓN

Rodriguez - Pereyra Coimbra - Zamudio - Alvarez - Christiani nato respecto a un grupo control y al grupo tratado con pasta a base de polvo de piedra pómez, similares a este estudio donde se observo que el spray de bicarbonato presentó valores significativos.

Según distintos estudios uno de ellos publicado por Osorio ${ }^{14}$ y colaboradores la mejor manera de limpiar el esmalte sería con un aparato que libera bicarbonato. Los autores consideran que las pastas de profilaxis producirían disminución de la energía superficial por el contenido en restos orgánicos ${ }^{14}$.

Sol-Segarra y col $^{15}$ analizaron diferentes sistemas de profilaxis siendo esta diferencia mayor en el grupo de spray con bicarbonato similar al encontrado en este estudio.

Para mejorar la adhesión y evitar fallos a este nivel es necesario que el esmalte tenga una energía superficial alta y el ácido y la resina adhesiva una humectabilidad también alta. En circunstancias normales el esmalte tiene una energía superficial baja esto le preserva su integridad estructural y además impide la adherencia bacteriana.

La contaminación de la superficie dentaria produciría cambios en la energía superficial afectando la adhesión. Según otros estudios 14 la mejor manera de limpiar el esmalte sería con el aparato de bicarbonato. Las pastas de profilaxis producirían disminución de la energía superficial por el contenido en restos orgánicos. La limpieza del esmalte con bicarbonato nos mejoraría la adhesión.

\section{Conclusión}

Entre los tres métodos de profilaxis se observó mayor resistencia en la resina reforzada en la que se empleó tratamiento previo con spray de bicarbonato mejorando la fuerza de adhesión, encontrándose diferencia significativa respecto al resto de los grupos.

Se sugiere un mayor número de estudios, realizando mediante pruebas en vivo y a largo plazo para poder valorar la eficacia de las diferentes profilaxis sobre la superficie dentaria.

\section{Bibliografía}

I. Anausavice, K. Ciencia de los materiales dentales de phillips. $10^{\circ}$ edición. ed. Macgraw-hill Interamericana.

2. Macchi, R. Materiales dentales. $3^{\circ}$ edición. ed. panamericana. 2000.

3. Perdigao J. Aadesao aos tecidos dentários. in: baratieri $\mathrm{nl}$ et al. Odontología restauradora. Fundamentos e possibilidades. Ia. edicao, Livraria Santos Editora. Sao Paolo 200I; 4: 83-128.

4. Suárez-quintanilla $\mathrm{J}$. Estudio del grabado ácido en las fisuras mediante microscopía electrónica de barrido. Rev esp odonto-estomatol 1987; 35: 467-74.

5. Scott $L$. The effect of an air-polishing device on sealant bond strength. Journal Proth dent 1987; 58: 384-7.

6. Huennekeus $S$ y col Effects of air-polishing on the abrasion of occlusal sealants. Quintessence int 1991; 22: 58I-5.

7. Puppin-rontani R. Caldo-teixeira A. Sinhoreti M. Etching time evaluation on the shear bond strength of two adhesive systems in primary teeth. Cienc Odontol Bras 20047 (3): 6-14.

8. Teixeira A, Rontani R. Eficácia da adesão em dentes decíduos: análise da literatura. Rev posgrad 2002; 9 (I): 70-4.

9. Cavalcanti, A y col Resistencia al cizallamiento de dos sistemas adhesivos autocondicionantes en dientes decíduos - estudio in vitro Acta odontol. venez. [online]. jun. 20।4: 46 (2).

10. Barceló S, Velásquez M, Guerrero lbarra J. Resistencia al desalojo por empuje de materiales restaurativos directos. Revista Odontológica Mexicana. 2005: 9 (4): I78-184.

II. Aboush Y, Tareen A, Elderton R. Resin to enamel bonds: effects of cleaning the enamel surface with prophylaxis pastes containing fluoride or oil. Br Dent J 1991; 171: 207-9.

12. Ray-Bogert T, García-Godoy F. Effect of prophylaxis agents on the shear bond strength of a fissure sealant. Pediatr Dent 1992; 14: 50-I.

13. Brown J, Barkmeier W. Comparison of six enamel treatment procedures for sealant bonding. Pediatr Dent 1996; 18: 29-31.

14. Osorio R, Toledano M. Adhesión en odontología. Arte y ciencia de los materiales Odontológicos. Madrid Ediciones Avances medico-dentales, s.l. 2000: |8|-2|6.

15. Sol-segarra E, Espasa-Suárez E, Boj-Quesada J. Influencia de diferentes sistemas de profilaxis en la adhesión de un sellador de fisuras al esmalte. RCOE 2005; 10 (2): 177- 182. 